\title{
Time-dependent Hartree-Fock and the one-body dissipation for head-on collisions
}

\author{
D. M. Brink \\ Theoretical Physics Department, University of Oxford, 1, Keble Road, Oxford, England \\ Fl. Stancu \\ Institut de Physique, University of Liège, Sart Tilman, 4000 Liège 1, Belgium \\ (Received 18 August 1980)
}

Trajectory calculations for head-on collisions of ${ }^{86} \mathrm{Kr}+{ }^{139} \mathrm{La}$ and ${ }^{84} \mathrm{Kr}+{ }^{209} \mathrm{Bi}$ are performed based on several nucleus-nucleus potentials and the window formula for friction. Results are compared to the time-dependent Hartree-Fock calculations from literature in the same energy range.

NUCLEAR REACTIONS Head-on classical trajectory calculations ${ }^{86} \mathrm{Kr}+{ }^{139} \mathrm{La}$
at $E_{1 \mathrm{ab}}=360-1000 \mathrm{MeV}$ and ${ }^{84} \mathrm{Kr}+{ }^{209} \mathrm{Bi}$ at $E_{1 \mathrm{ab}}=500-1000 \mathrm{MeV}$.

\section{INTRODUCTION}

The aim of the present study is to get more insight into the one-body aspect of the dissipative mechanism of heavy-ion collisions. For this purpose we make trajectory calculations based on the window formula ${ }^{1,2}$ and compare the results with time-dependent-Hartree-Fock (TDHF) calculations $^{3,4}$ for head-on collisions. The window formula $^{2}$ and the time-dependent-Hartree-Fock meth$\mathrm{od}^{5}$ are based on the nearly independent motion of nucleons in a deformed container and in a timedependent mean field, respectively. In other respects, however, they are different. The window formula is based on a classical perturbative treatment; the TDHF is based on a self-consistent quantal description. Although one hopes to get a better understanding of the dissipation mechanism of colliding nuclei by comparing the two methods, it is difficult to relate them theoretically. ${ }^{6}$ We therefore compare the results of the two approaches. A similar idea is contained in Ref. 7, which includes in addition an extra excitation mechanism of the collective surfaces modes.

The trajectory calculations we present in this paper are for head-on collisions of the system ${ }^{86} \mathrm{Kr}+{ }^{139} \mathrm{La}$ and ${ }^{84} \mathrm{Kr}+{ }^{209} \mathrm{Bi}$, as in the TDHF calculations of Refs. 3 and 4 and the results are compared in the same energy range. The friction is given by the window formula and we make several choices for the conservative force. Some preliminary results have been presented elsewhere. ${ }^{8}$

In Sec. II, we summarize the TDHF results. In Sec. III, we present our trajectory calculations and discuss them in relation to the TDHF results. In the last section we draw some conclusions about the role of the conservative and nonconservative forces.

\section{DESCRIPTION OF THE TDHF RESULTS}

An important question in the TDHF calculations ${ }^{3}$ was to see if heavy ion systems with composite mass greater then 200 undergo fusion. It turned out that the threshold behavior was rather complicated and is different from the present classical trajectory calculations, as we shall see below.

${ }^{86} \mathrm{Kr}+{ }^{139} \mathrm{La}$. T DHF results for head-on collisions show two regimes of fusionlike behavior: a narrow region just above the Coulomb barrier at $E_{\mathrm{ab}} \sim 410 \mathrm{MeV}$ and another broader region between $E_{1 \mathrm{ab}} \simeq 650$ and $850 \mathrm{MeV}$. For energies below $650 \mathrm{MeV}$, except around $E_{1 \mathrm{ab}} \simeq 410 \mathrm{MeV}$, the projectile is reflected from the target, and beyond $850 \mathrm{MeV}$ it "passes through" the target. In all these cases the final kinetic energy has an almost constant value of $195 \pm 5 \mathrm{MeV}$.

${ }^{84} \mathrm{Kr}+{ }^{209} \mathrm{Bi}$. For this system TDHF calculations show only one fusion region between $E_{1 \mathrm{ab}} \simeq 850$ $\mathrm{MeV}$ and $E_{1 \mathrm{ab}} \simeq 1100 \mathrm{MeV}$. For $E_{1 \mathrm{ab}}<850 \mathrm{MeV}$ the projectile "bounces" off the target while for $E_{1 \mathrm{ab}}>1100 \mathrm{MeV}$ it passes "through." In the region just above the barrier some very long-lived configurations have been seen. According to Ref. 3 such dynamical resonances recall the fusionlike behavior which was found just above the Coulomb barrier for the ${ }^{86} \mathrm{Kr}+{ }^{139} \mathrm{La}$ system.

Another quantity of interest in head-on collisions is the stopping time $t_{s}$. We define it as the interval from the instant when the nuclear surfaces touch $(s=0)$ to the instant of closest approach. Such stopping times are extracted from the TDHF calculations ${ }^{4}$ and are indicated in Table I as a function of the bombarding energy .

As Fig. 1 of Ref. 3 suggests, the nuclei approach each other very fast. They slow down and then remain for a period at an almost constant mini- 
TABLE I. Stopping times $t_{s}$ in units $10^{-22} \mathrm{~s}$ as a function of the bombarding energy $E_{\text {lab }}$ for ${ }^{84} \mathrm{Kr}+{ }^{209} \mathrm{Bi}$. The second column indicates values of $t_{s}$ extracted from Ref. 4 and column 3 gives the stopping time obtained with the standard proximity potential.

\begin{tabular}{ccc}
\hline \hline & $==0=$ \\
$E_{\text {lab }}$ & $t_{s}^{\mathrm{TDHF}}$ & $t_{s}^{\mathrm{SP}}$ \\
\hline & & \\
500 & 4.9 & 5.2 \\
550 & 4.0 & 4.4 \\
600 & 3.4 & 4.0 \\
714 & 3.0 & 3.4 \\
800 & 2.5 & 3.0 \\
1000 & 2.1 & 2.8
\end{tabular}

mum separation distance. In estimating the stopping time we consider the first instant when the relative motion appears to have stopped, and therefore the values of $t_{s}$ given in Table I might be underestimated. One can see that the stopping time is of the order of a few units of $10^{-22} \mathrm{~s}$ and that it is shorter for higher energies. In the next section we shall make a comparison with the stopping times resulting from classical trajectory calculations.

\section{CLASSICAL TRAJECTORY CALCULATIONS}

In this section we write an equation for the relative motion of the two heavy ions in a head-on collision. We assume that the nuclei retain their spherical shape throughout the collision, that the mass associated with the relative motion is just the reduced mass $\mu$, and the force acting between the two nuclei has a conservative part and a frictional part.

If we call $s$ the separation between the surfaces of the interacting nuclei, the equation to be solved in head-on collisions is

$$
\mu \frac{d^{2} s}{d t^{2}}=-\frac{d V}{d s}-2 N(s) \frac{d s}{d t} .
$$

On the right-hand side (rhs) the first term is the conservative force derived from the potential $V(s)$ discussed below. The second term is the radial component of the window friction formula ${ }^{2}$ defined as the product of twice the radial velocity $d s / d t$ and the total flux $N(s)$ passing through the window, for which we use the analytic expression of Randrup. ${ }^{9}$

Four different choices have been made for $V(s)$ :

1. Pure Coulomb potential for overlapping charge distributions using the formula of Bondorf et al. ${ }^{10}$

2. Coulomb (Bondorf) + nuclear potential of Siwek-Wilczyńska and Wilczyński ${ }^{11}$ (SW-W).

3. Coulomb (Bondorf) + standard proximity potential $^{12}$ (SP).

4. Coulomb (Bondorf) + modified proximity potential of Randrup ${ }^{13}$ (MP).

Figures 1 and 2 show the various potentials for ${ }^{86} \mathrm{Kr}+{ }^{139} \mathrm{La}$ and ${ }^{84} \mathrm{Kr}+{ }^{209} \mathrm{Bi}$. They are given as a function of the separation distance $R$ between the nuclear centres related to $s$ by

$$
R=s+C_{1}+C_{2},
$$

where

$$
C_{i}=R_{i}-\frac{b^{2}}{R_{i}} \quad(i=1,2),
$$

with

$$
b=1 \mathrm{fm} .
$$

For each potential the nuclear radii $R_{i}$ are taken according to the corresponding definition given either in Ref. 11 or 12 . But in fact the numerical values of $C_{i}$ are almost the same for the three potentials. These give $C_{1}+C_{2} \simeq 10.7 \mathrm{fm}$ for ${ }^{86} \mathrm{Kr}$ $+{ }^{139} \mathrm{La}$ and $C_{1}+C_{2} \simeq 11.8 \mathrm{fm}$ for ${ }^{84} \mathrm{Kr}+{ }^{209} \mathrm{Bi}$. The standard proximity potential has a shallow pocket of $\sim 5.5 \mathrm{MeV}$ deep with respect to the barrier for ${ }^{86} \mathrm{Kr}+{ }^{139} \mathrm{La}$. For ${ }^{84} \mathrm{Kr}+{ }^{209} \mathrm{Bi}$ the potential is even shallower, the pocket being only $\sim 2 \mathrm{MeV}$ deep. In

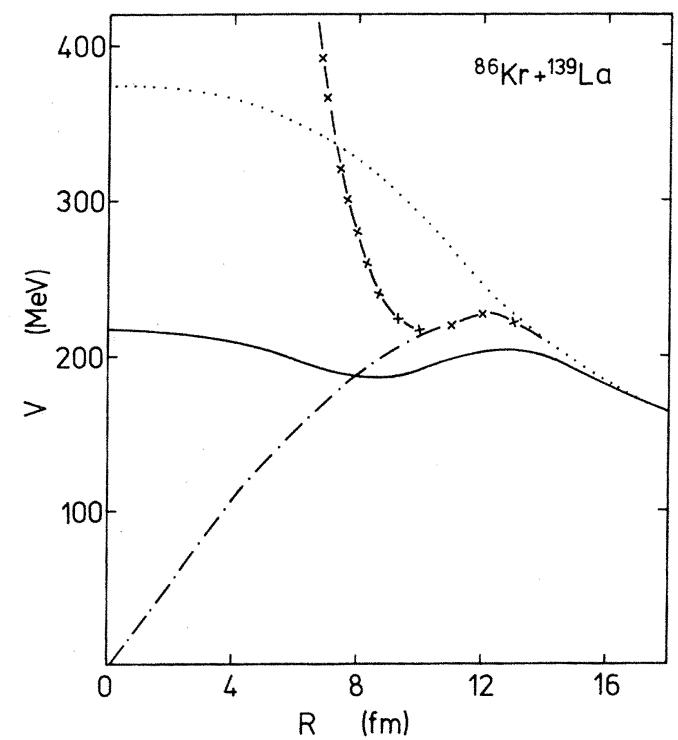

FIG. 1. Interaction potential of ${ }^{86} \mathrm{Kr}+{ }^{139} \mathrm{La}$ as a function of the separation distance between nuclear centers (...) - Coulomb, (-) -SW-W+ Coulomb, $(\mathrm{x}-\mathrm{x}-\mathrm{x})-\mathrm{SP}+$ Coulomb, (•-.-.) - MP+Coulomb. 


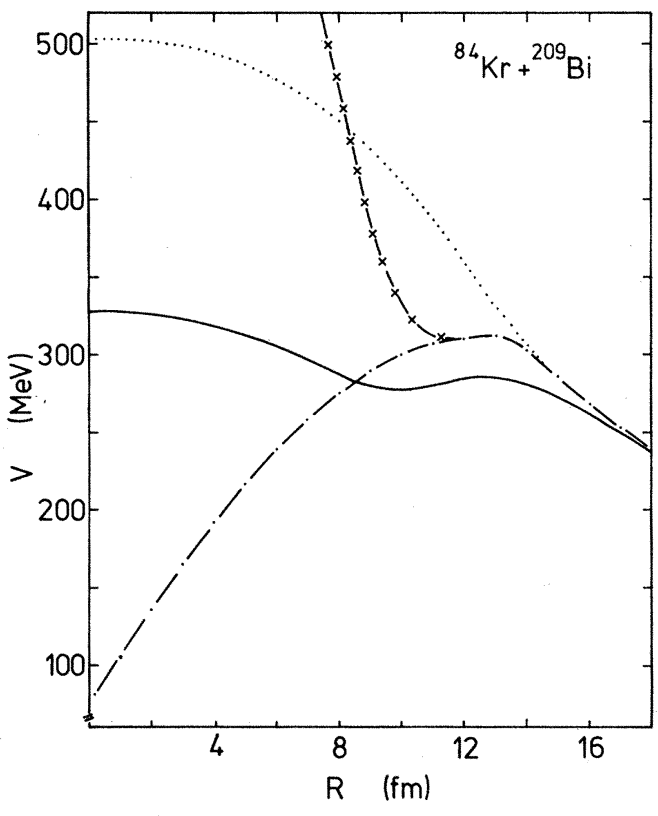

FIG. 2. Same as Fig. 1, but for ${ }^{84} \mathrm{Kr}+{ }^{209} \mathrm{Bi}$.

both cases the SW-W potential has a deeper pocket than the proximity potential. Typical for the modified proximity potential is a broad barrier beyond which the potential decreases linearly towards the center.

In Figs. 3 and 4 we show results of Eq. (1) solved with the initial conditions $s(0)=3.2 \mathrm{fm}$ and

$$
\left.\frac{d s}{d t}\right|_{t=0}=\left[2\left(E_{\mathrm{c} . \mathrm{m} .}-V(3.2)\right) / \mu\right]^{1 / 2}
$$

for ${ }^{86} \mathrm{Kr}+{ }^{139} \mathrm{La}$ and ${ }^{84} \mathrm{Kr}+{ }^{209} \mathrm{Bi}$, respectively. The initial point is chosen because the friction

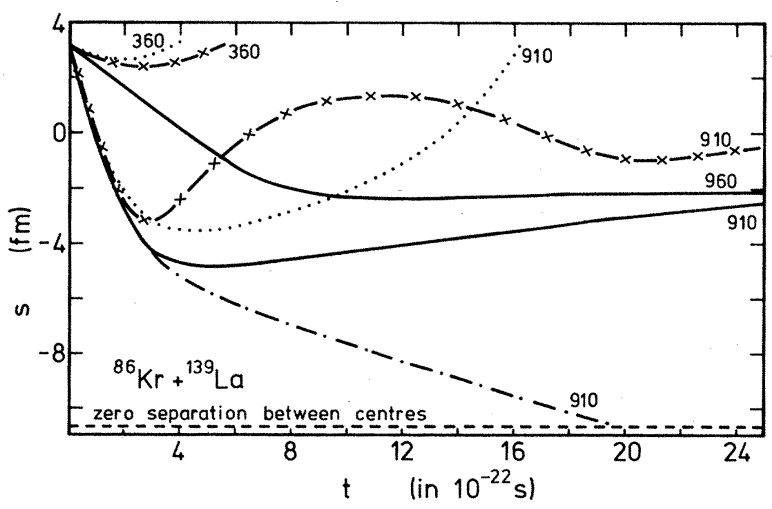

FIG. 3. Trajectories for ${ }^{86} \mathrm{Kr}+{ }^{139} \mathrm{La}$ at $E_{1 \mathrm{ab}}=360 \mathrm{MeV}$ and $E_{\mathrm{lab}}=910 \mathrm{MeV}$ for the potentials of Fig. 1 (same legend). The separation distance $s$ between surfaces is $s=R-10.70$ for $\mathrm{SW}-\mathrm{W}$ and $s=R-10.73$ for SP or MP potentials.

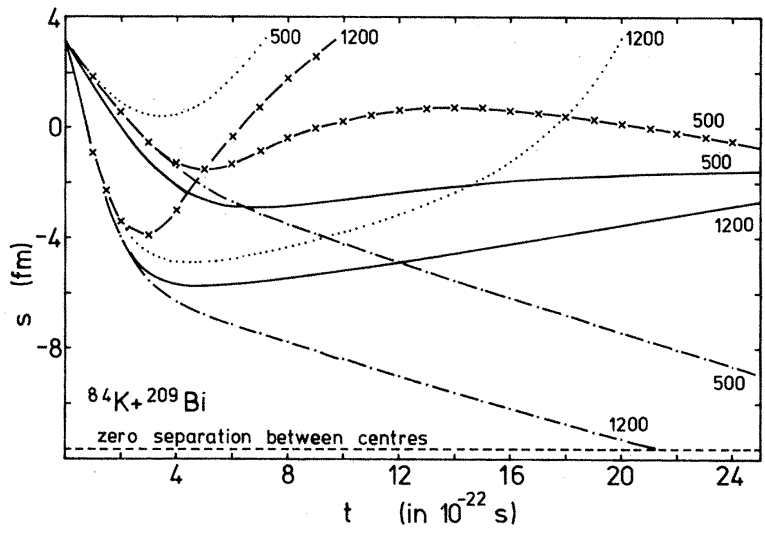

FIG. 4. Trajectories for ${ }^{84} \mathrm{Kr}+{ }^{209} \mathrm{Bi}$ at $E_{1 \mathrm{ab}}=500 \mathrm{MeV}$ and $E_{1 \mathrm{ab}}=1200 \mathrm{MeV}$ for the potentials of Fig. 1 (same legend). The separation distance $s$ between surfaces is $s=R-11.63$ for $\mathrm{SW}-\mathrm{W}$ and $s=R-11.66$ for $\mathrm{S} \mathrm{P}$ or $\mathrm{MP}$ potentials.

starts to act at $s=3.2 \mathrm{fm} .{ }^{9}$ Calculations were done in the same energy range as for the TDHF study. ${ }^{3,4}$ For each potential we show results for the lowest and highest values of the energy considered.

${ }^{86} \mathrm{Kr}+{ }^{139} \mathrm{La}$. Most of our discussion will concern the standard proximity potential. With the other potentials the results are qualitatively independent of energy as one can see from Fig. 3. In particular for the pure Coulomb the fragments always separate, for the $\mathrm{SW}-\mathrm{W}$ potential the projectile is always captured in the pocket and in the MP potential the projectile always goes to the center.

With the proximity potential we obtain a large fusion window from just above the barrier $\left(E_{\text {lab }} \simeq 370 \mathrm{MeV}\right)$ until about $1000 \mathrm{MeV}$, much wider that the lower fusion region in the TDHF calculations. Below and above this region the projectile bounces back. In the fusion region found in our calculations we observe oscillations in $s$ as a function of time, the amplitude of which increases with the bombarding energy.

${ }^{84} \mathrm{Kr}+{ }^{209} \mathrm{Bi}$. In the energy range $500-1200 \mathrm{MeV}$, none of the considered potentials gives the behavior observed in TDHF calculations. As Fig. 4 shows, the projectile is captured in the pocket of the SW-W potential or near the center of the MP potential over the whole energy range. The SP potential gives a fusion window from relative energies just above the barrier or $E_{1 \mathrm{ab}} \sim 440 \mathrm{MeV}$ until about $E_{1 \mathrm{ab}} \sim 700 \mathrm{MeV}$. This type of fusion is more reminiscent of the low energy fusionlike behavior seen in TDHF calculation of ${ }^{86} \mathrm{Kr}+{ }^{139} \mathrm{La}$, and it would correspond to the long-lived configurations found at $E_{1 \mathrm{ab}}=510$ and $525 \mathrm{MeV}$ in the T DHF studv of ${ }^{84} \mathrm{Kr}+{ }^{209} \mathrm{Bi}$. In our calculations 
the fusion appears as a combined effect of the existence of a shallow pocket in the SP potential and the strong character of the window friction. For both systems ${ }^{86} \mathrm{Kr}+{ }^{139} \mathrm{La}$ and ${ }^{84} \mathrm{Kr}+{ }^{209} \mathrm{Bi}$ we find some similarity between trajectory calculations and the TDHF results. All the potentials we use, except the MP potential, stop the projectile. The stopping times, as defined in the previous section, are sensitive to the conservative force. For ${ }^{84} \mathrm{Kr}+{ }^{209} \mathrm{Bi}$ the values we found are comparable but somewhat larger than the TDHF stopping times. Except for the Coulomb potential, it also decreases with the energy like in TDHF calculations. The nearest to $\mathrm{TDHF}$ results are those of the SP potential and they are also indicated in Table I. The difference between TDHF and trajectory calculations essentially appears after the instant of closest approach.

\section{CONCLUSIONS}

The trajectory calculations include the effects of both the conservative and the nonconservative forces. Therefore we can at most draw some qualitative conclusions about the dissipation.

For high energies the slowing down of the relative motion in the approach phase is initially due to the frictional force, but in the last stages of the slowing down process the conservative force makes a difference. At lower energies the frictional force is not so dominant. The conservative force is more important at all stages and makes a difference to the stopping times and stopping distances. The stopping times calculated using the standard proximity potential are consistent with the results of the TDHF calculations.

In the late stages of the collision our trajectory calculations give quite different results from TDHF. Calculations with the standard proximity potential lead to fusion except at very high incident energies. The modified proximity and the SW-W potential always give fusion when the incident energy is about the Coulomb barrier. An important reason for this difference is that the nuclei are constrained to be spherical in our trajectory calculations. We do not allow other degrees of freedom such as a neck formation to develop in the separation phase of the reaction. Swiatecki ${ }^{14}$ has shown that neck formation gives rise to important changes in the potential energy landscape, and is crucial in deciding whether the nuclei separate again after the collision. According to the model of Ref. 14 the value of the "effective fissility" parameter

$$
X=\frac{3 Z_{1} Z_{2} e^{2}}{10 \pi \gamma\left(R_{1}+R_{2}\right) R_{1} R_{2}}, \quad \gamma \simeq 1 \mathrm{MeV} \mathrm{fm}^{-2}
$$

is important for determining whether the nuclei reseparate. If $X>0.57$ the nuclei should separate unless the incident energy is sufficiently high, while if $X<0.57$, the composite system should normally fuse. In our examples $X \simeq 0.81$ for ${ }^{86} \mathrm{Kr}$ $+{ }^{139} \mathrm{La}$ and $X \simeq 0.97$ for ${ }^{84} \mathrm{Kr}+{ }^{209} \mathrm{Bi}$. The results of the TDHF calculations are consistent with this picture in both cases.

From this discussion it seems reasonable to conclude that a more complex description of classical trajectory calculations would be necessary in order to cover certain aspects of TDHF results. A possibility is to include more deg rees of freedom, in particular a neck radius. Another possibility is to introduce a time or energy dependent potential. An interesting problem would be to study the relation between these procedures.

This work has been supported by NATO Research Grant No. 1782.
${ }^{1}$ W. J. Swiatecki, J. Phys. (Paris) $\underline{33}$, Suppl. 8-9, C5 (1972).

${ }^{2}$ J. Blocki, Y. Boneh, J. R. Nix, J. Randrup, M. Robel, A. J. Sierk, and W. J. Swiatecki, Ann. Phys. (N. Y.) 113,330 (1978).

${ }^{3}$ K. T. R. Davies, K. R. Sandhya Devi, and M. R. Strayer, Phys. Rev. Lett. $\underline{44}, 23$ (1980).

${ }^{4} \mathrm{~K}$. T. R. Davies et al., private communication.

${ }^{5}$ P. Bonche, S. E. Koonin, and J. Negele, Phys. Rev. C 13, 1226 (1976).

${ }^{6} \mathrm{~S}$. E. Koonin, Lecutres given at the International School of Physics "Enrico Fermi," Varenna, Italy, 1979.

${ }^{7}$ R. A. Broglia, A. K. Dhar, H. Esbensen, B. S. Nilsson, and C. H. Dasso, Phys. Lett. $\underline{83 \mathrm{~B},} 301$ (1979).
${ }^{8}$ D. M. Brink and Fl. Stancu, International Workshop on Gross Properties of Nuclei and Nuclear Excitations VIII, Hirschegg, Austria, edited by H. Feldmeier (Technische Hochschule Darmstadt, Darmstadt, 1980), p. 162.

$9^{9}$ J. Randrup, Ann. Phys. (N. Y.) 112, 356 (1978).

${ }^{10}$ J. P. Bondorf, M. I. Sobel, and D. Sperber, Phys. Rep. C15, 83 (1974).

${ }^{11} \mathrm{~K}$. Siwek-Wilczyńska and J. Wilczyński, Phys. Lett. 74B, 313 (1978).

${ }^{12}$ J. Blocki, J. Randrup, W. J. Swiatecki, and C. F. Tsang, Ann. Phys. (N. Y.) 105, 427 (1977).

${ }^{13}$ J. Randrup, Nucl. Phys. A307, 319 (1978).

${ }^{14}$ W. J. Swiatecki, Phys. Scr. (to be published). 